\section{QUALIDADE DE VIDA DE PACIENTES ONCOLÓGICOS EM CUIDADOS PALIATIVOS}

\section{QUALITY OF LIFE OF ONCOLOGICAL PATIENTS UNDER PALLIATIVE CARE}

\section{CALIDAD DE VIDA DE LOS PACIENTES ONCOLÓGICOS EN CUIDADOS PALIATIVOS}

Jaqueline Fantini Figueiredo ${ }^{1}$, Vitor Marques Souza ${ }^{1}$, Hiago Victor Coelho ${ }^{1}$, Raissa Silva Souza ${ }^{2}$

\section{RESUMO}

Objetivo: Avaliar a qualidade de vida de pacientes oncológicos em cuidados paliativos, por meio de instrumento validado. Método: Trata-se de um estudo descritivo, quantitativo com pacientes internados em uma unidade de oncologia de um hospital de grande porte do Centro-Oeste de Minas Gerais. Resultado: observou-se uma perda importante na qualidade de vida dos participantes, em especial no domínio de "bem-estar emocional" e "bem-estar funcional". Contudo, os domínios de "bem-estar físico" e "bem-estar social-familiar" foram bem avaliados pelos participantes. Conclusão: A equipe de saúde, em especial a de enfermagem, necessita se instrumentalizar para a identificação e resolução oportuna de questões que podem trazer prejuízo às diferentes dimensões da vida da pessoa com câncer, atentando para a manutenção do controle dos sintomas, fomentando a participação da família nos cuidados e fornecendo o suporte emocional e funcional adequados às demandas individuais da pessoa sob cuidados paliativos.

Descritores: Qualidade de vida; Cuidados paliativos; Enfermagem; Oncologia.

\section{ABSTRACT}

Objective: to evaluate the quality of life of cancer patients under palliative care using a validated instrument. Method: this is a descriptive, quantitative study with patients admitted to an oncology unit of a large hospital in the Midwest region of Minas Gerais. Result: there was a significant loss in the participants' quality of life, especially in the domains "emotional well-being" and "functional well-being". However, the domains "physical well-being" and "social-family well-being" were well evaluated by the participants. Conclusion: the health team, especially the nursing team, needs to be skilled to identify and timely solve issues that can harm the different dimensions of the person with cancer, with a view to maintaining symptom control, fostering the family participation in the care and providing the emotional and functional support appropriate to the individual demands of the person under palliative care.

Descriptors: Quality of life; Palliative care; Nursing; Oncology.

\section{RESUMEN}

Objetivo: Evaluar la calidad de vida de pacientes oncológicos en cuidados paliativos por medio de instrumento validado. Método: Se trata de un estudio descriptivo, cuantitativo con pacientes internados en una unidad de oncología de un hospital de gran porte del Centro-Oeste de Minas Gerais. Resultado: se observó una pérdida importante en la calidad de vida de los participantes, en particular en el ámbito del "bienestar emocional" y el "bienestar funcional". Sin embargo, los ámbitos de "bienestar físico y bienestar social-familiar" fueron bien evaluados por los participantes. Conclusión: El equipo de salud, en especial la de enfermería, necesita instrumentalizarse para la identificación y resolución oportuna de cuestiones que pueden traer perjuicio a las diferentes dimensiones de la vida de la persona con cáncer, atentando para el mantenimiento del control de los síntomas, fomentando la participación de la familia en el cuidado y proporcionando el soporte emocional y funcional adecuado a las demandas individuales de la persona bajo cuidados paliativos.

Descriptores: Calidad de vida; Cuidados paliativos; Enfermería; Oncológica.

${ }^{1}$ Graduado em Enfermagem pela Universidade Federal de São João del-Rei. ${ }^{2}$ Graduada em Enfermagem. Doutora em Enfermagem pela Universidade Federal de Minas Gerais. Docente na Universidade Federal de São João del-Rei.

Como citar este artigo:

Figueiredo JF, Souza VM, Coelho HV, et al. Qualidade de Vida de Pacientes Oncológicos em Cuidados Paliativos. 2018;8:e2638. [Access__]; Available in: DOI: http://dx.doi.org/10.19175/recom.v8i0.2638 


\section{INTRODUÇÃO}

O câncer constitui um importante problema de saúde pública mundial, sendo a principal causa de morte em países em desenvolvimento, como é o caso do Brasil. No contexto nacional, o câncer ocupa a segunda posição no ranking de mortes por causas diversas da população geral, sendo que, para o biênio de 2016-2017, há projeção de que cerca de 600 mil novos casos da doença serão diagnosticados ${ }^{(1-2)}$.

O diagnóstico de câncer repercute de modo importante na vida da pessoa e de sua família, em especial quando a doença se apresenta em estágio avançado e com metástases, fora de possibilidade terapêutica de cura. Quando isso acontece, diz-se que a pessoa se encontra em cuidados paliativos (CP), sendo que, a partir desse ponto, a ênfase recai sobre as medidas que visam a identificação, a avaliação e o tratamento oportuno de sintomas físicos, psicossociais e espirituais que repercutem na qualidade de vida (QV) da pessoa ${ }^{(3-4)}$.

Desse modo, os CP podem ser entendidos como uma abordagem terapêutica ao paciente com doença fora de possibilidade de cura que, por meio de intervenções direcionadas ao alívio dos sintomas e à prevenção do sofrimento físico, psicossocial e espiritual, visa à $\mathrm{QV}^{(3)}$. Para tanto, são desenvolvidas diferentes abordagens assistências pelos integrantes das equipes dos serviços de saúde da atenção primária, secundária e terciária, quais sejam, a equipe de enfermagem, médica, nutrição, serviço social, psicologia, terapia ocupacional, dentre outras.

$\mathrm{O}$ alvo das equipes de CP é a otimização da QV da pessoa, sendo que, em se tratando desta última, não há consensos sobre uma definição universal. No entanto, existe consonância sobre seu caráter multidimensional, subjetivo e individual concernentes tanto à saúde, envolvendo questões de ordem física, funcional, emocional e mental, quanto à elementos importantes da vida das pessoas como trabalho, família, amigos, e outras circunstâncias do cotidiano. A QV também é influenciada pelas percepções da pessoa sobre sua posição na vida em relação à cultura, sistema de valores, objetivos pessoais e expectativas, não tendo relação apenas com a presença ou ausência de doença ${ }^{(5)}$.

A avaliação da QV resulta da análise da percepção do indivíduo em relação à sua posição na vida, tanto no contexto da cultura e dos sistemas de valores nos quais vive, como em relação aos seus objetivos, expectativas, padrões e preocupações individuais em relação à saúde, à doença e ao que traz valor e significado para a vida da pessoa ${ }^{(5)}$. Informações sobre a QV de grupos específicos de pessoas podem ser úteis no monitoramento de diversas intervenções terapêuticas, de diferentes naturezas, favorecendo a tomada de decisão clínica e cuidados em saúde ${ }^{(5-6)}$.

Em relação a casos de câncer avançado fora de possibilidade terapêutica de cura, sabe-se que vários fatores podem comprometer a QV, desde o próprio diagnóstico da doença até os efeitos tóxicos das terapias empregadas, as limitações impostas pela doença e pelo tratamento e o desenvolvimento de condições clínicas associadas à ansiedade e a depressão ${ }^{(4)}$. Nesse sentido, entende-se que a avaliação da QV dessas pessoas, por meio de instrumentos específicos para tanto, seja essencial para o planejamento e implementação de planos de cuidados que potencializem seu bem-estar e satisfação com a vida ${ }^{(4-6)}$.

A utilização de instrumentos de mensuração de resultados dos CP é considerada essencial para a avaliação da qualidade do cuidado oferecido, aumentando o conhecimento sobre a experiência do paciente com câncer e com as terapias empregadas, além de fornecer informações sobre a eficiência das intervenções propostas, tendo em vista a melhoria dos sintomas e da QV das pessoas. Dentre as possibilidades de mensuração de resultados em $\mathrm{CP}$, a avaliação da QV tem se tornado cada vez mais importante na compreensão da repercussão das intervenções propostas pelas equipes assistenciais na vida delas ${ }^{(5-6)}$.

Diferentes instrumentos de mensuração da QV foram traduzidos e validados para o Brasil, com escalas específicas para a avaliação da qualidade de vida em pessoas sob cuidados paliativos $^{(7-10)}$. Contudo, tais instrumentos são pouco utilizados enquanto ferramenta de avaliação sistemática dos pacientes na prática clínica, restringindo seu uso às pesquisas ${ }^{(7)}$.

Acredita-se que a aplicação de escalas validadas para a mensuração da QV, em diferentes perfis de pacientes e em diferentes contextos assistenciais, possa trazer pistas sobre as necessárias adequações a serem realizadas na conformação dos planos assistenciais em contextos distintos de assistência à saúde. Nesse sentido, o presente estudo tem por objetivo avaliar a qualidade de vida de pacientes em 
cuidados paliativos internados em uma unidade de oncologia de um hospital de grande porte do Centro-Oeste mineiro, por meio da aplicação de instrumento validado.

\section{METódo}

Trata-se de um estudo descritivo, de abordagem quantitativa, desenvolvido com pacientes em CP internados em uma unidade de oncologia de um hospital de grande porte credenciado na Unidade de Alta Complexidade em Oncologia (UNACON). A assistência oferecida na instituição inclui as modalidades integradas de diagnóstico, cirurgia oncológica, radioterapia, quimioterapia, oncologia clínica, hematologia, medidas de suporte, não existindo, até o término da presente pesquisa, a modalidade de cuidados paliativos. $O$ referido hospital é referência para o tratamento de pacientes portadores de câncer de 86 cidades da macrorregião Centro-Oeste de Minas Gerais ${ }^{(11)}$.

A unidade oncológica onde o estudo foi realizado é destinada à internação clínica de pessoas com câncer em distintos estágios e com diferentes prognósticos, vindas tanto do domicílio, com agudização de problemas decorrentes da doença e/ou do tratamento, como das unidades de tratamento cirúrgico, quimioterápico e radioterápico da instituição.

De modo geral, os pacientes com indicação de $C P$, internados nessa unidade, encontram-se com doença avançada, em estado progressivo de declínio, com capacidade funcional limitada, inviabilizando sua participação na pesquisa. Além disso, a rotatividade desses pacientes na unidade é relativamente baixa, pois muitos preferem permanecer internados devido à inexistência de serviços de apoio e cuidados no nível domiciliar na região.

Considerando essas especificidades, a composição da amostra foi não-probabilística, por conveniência, tendo sido composta por pacientes com indicação médica de $\mathrm{CP}$, entre os meses de abril e junho de 2017.

Os critérios de inclusão foram: estar internados no setor oncológico do hospital; ter definição médica expressa no prontuário de CP; ter idade superior a 18 anos e estar lúcidos e em condições de se comunicar verbalmente. Os critérios de exclusão foram: pacientes com capacidade funcional igual ou menor a $30 \%$ (estágio terminal), avaliados pela escala Palliative Performance Scale (PPS), versão $2^{(12)}$.

Os dados foram coletados por meio de entrevistas individuais, sendo que os pacientes elegíveis foram abordados individualmente, no próprio quarto onde estavam internados, considerando as limitações físicas para o deslocamento do leito. Para a coleta dos dados, foram utilizados dois instrumentos, quais sejam: o primeiro contendo as variáveis sociodemográficas e clínicas e o segundo, contendo as variáveis relacionadas à QV.

$O$ instrumento de QV escolhido foi o FACIT - Pal, desenvolvido pelo grupo Functional Assessment of Chronic IIIness Terapy (FACIT), responsável pela construção e divulgação de instrumentos de $Q V$ voltados a pacientes com doenças e agravos não transmissíveis. O FACIT-Pal é amplamente usado nos sistemas de saúde norte-americanos, com alta consistência interna, confiabilidade e validade, sendo um instrumento voltado para a mensuração da qualidade de vida de pessoas com doença avançada ou limitante da vida ${ }^{(13)}$, tendo sido traduzido para o idioma português pelo grupo FACIT e, posteriormente, validado culturalmente ${ }^{(9)}$.

O FACIT-Pal é composto por uma escala geral de avaliação de Qualidade de Vida (FACT-G), combinada com uma subescala específica para a avaliação de pessoas portadoras de doenças fora de possibilidade terapêutica de cura e que requeriam cuidados paliativos. $O$ instrumento inclui a avaliação de cinco domínios, por meio de 41 perguntas, quais sejam: "bem-estar físico"; "bem-estar social/familiar"; "bem-estar emocional"; "bem-estar funcional" e "preocupações adicionais". As repostas são graduadas em uma escala do tipo Liket com 5 opções que vão desde a resposta "nada" até "demais" "13).

A subescala específica para a avaliação de pessoas portadoras de doenças fora de possibilidade terapêutica de cura contém 19 itens com informações referentes: aos sintomas mais prevalentes em CP (p.ex. falta de ar, prisão de ventre, xerostomia), à relação com a família e os amigos (percepção de apoio social), a questões relacionadas com o fechamento da vida (p. ex., "ter feito as pazes com os outros", "sentindo-se esperançoso", "fazendo com que cada dia seja importante"), à tomada de decisão, comunicação e às habilidades (por exemplo, capacidade de tomar decisões, de pensar com clareza, e discutir abertamente preocupações) ${ }^{(13)}$.

Para a mensuração da qualidade de vida, foram realizados cálculos dos escores de cada domínio. O escore dos domínios de "bem-estar físico", "bem-estar social/familiar" e "bem-estar funcional" varia de 0 a 28 pontos, sendo que 0 
escore do domínio "bem-estar emocional" varia de 0 a 24 pontos e o escore do domínio de "preocupações adicionais" varia de 0 a 76 pontos. Quanto maior a pontuação obtida, melhor a avaliação da qualidade de vida referente àquele domínio ${ }^{(13)}$.

Os autores da FACIT-Pal apresentam três índices que devem ser calculados, quais sejam: (1) o Índice dos Resultados do Teste (Trial Outcome Index TOI), obtido pelo somatório dos domínios "bem-estar físico", "bem-estar funcional" e "preocupações adicionais", sendo que a pontuação desse escore varia de 0 a 132; (2) o Índice de Qualidade de Vida Geral (FACT-G), obtido pelo somatório dos domínios "bem-estar físico", "bem-estar social/familiar", "bem-estar emocional" e "bem-estar funcional", sendo que a pontuação desse escore varia de 0 a 108, e (3) o Índice total da escala de Qualidade de Vida nas Pessoas sob Atenção Paliativa (FACIT-Pal), obtido pelo somatório de todos os domínios da escala ("bem-estar físico"; "bem-estar social/familiar"; "bem-estar emocional"; "bem-estar funcional" e "preocupações adicionais"), com valor variando entre 0 a 184 pontos. Quanto maiores as pontuações nos escores, melhor a percepção da pessoa sobre sua qualidade de vida ${ }^{(13)}$.

Utilizou-se o programa Statistical Package for the Social Sciences (SPSS), versão 22.0, para o tratamento e análise dos dados. Procedeu-se à análise descritiva dos dados apresentados por meio das medidas de tendência central (mediana), frequência absoluta, medidas de dispersão (máximo e mínimo) e percentis.

A participação na pesquisa foi voluntária, mediante assinatura do Termo de Consentimento Livre e Esclarecido. O protocolo do estudo foi aprovado pelo Comitê de Ética em Pesquisa da Universidade Federal de São João del-Rei, CAAE $\mathrm{n}^{\circ}$ 0043.0.410.20310 e pelo Comitê de Ética da Instituição, CAAE n0043.0.410.203-10A, conforme Resolução 466/12 do Conselho Nacional de Saúde.

\section{RESULTADOS E DISCUSSÃO}

A amostra foi composta, majoritariamente, por pessoas do sexo feminino $(n=5 ; 55,6 \%)$, casadas $(n=5 ; 55,6 \%)$, com filhos $(n=7 ; 77,8 \%)$, sendo os filhos apontados como um dos principais cuidadores pelos participantes. A maioria deles tinha baixa escolaridade, predominando os participantes que possuíam, como maior grau de instrução formal, o ensino fundamental incompleto $(n=3 ; 33,3 \%)$ e ensino fundamental completo $(n=2 ; 22,2 \%)$. Dados semelhantes foram encontrados em estudo(14) com 124 pacientes em CP, no qual, a maioria dos participantes era do sexo feminino, casada e com baixo nível de ensino. Estes dados podem evidenciar que, mesmo com diversas campanhas e políticas públicas voltadas para o público feminino, continua elevado o contingente de pessoas desse sexo. Um dos fatores que podem contribuir para tanto é a baixa escolaridade, que é apontada, na literatura, como uma variável que retarda a identificação do câncer e o início do tratamento, por dificultar o acesso e a assimilação das informações sobre as medidas de prevenção, diagnóstico, controle e tratamento da doença.

As frequências e percentuais referentes aos demais dados sociodemográficos da amostra do presente estudo encontram-se apresentadas na Tabela 1.

Tabela 1 - Caracterização do perfil sociodemográfico dos pacientes oncológicos em Cuidados Paliativos. Divinópolis, MG, mar.-jun., 2017 ( $n=9$ ).

\begin{tabular}{|c|c|c|c|}
\hline Variável & Distribuição em categorias & $\mathbf{N}$ & $\%$ \\
\hline \multirow[t]{5}{*}{ Autodenominação racial } & Mulato & 2 & 22,2 \\
\hline & Pardo & 3 & 33,3 \\
\hline & Negro & 2 & 22,2 \\
\hline & Branco & 0 & 0,0 \\
\hline & Não declarado & 2 & 22,2 \\
\hline \multirow[t]{4}{*}{ Faixa Salarial } & Até um salário mínimo & 5 & 55,6 \\
\hline & 1 a 3 salários & 3 & 33,3 \\
\hline & $\geq 4$ salários & 0 & 0,0 \\
\hline & Não declarado & 1 & 11,1 \\
\hline \multirow[t]{5}{*}{ Renda Familiar } & Até um salário mínimo & 0 & 0,0 \\
\hline & 1 a 3 salários & 6 & 66,7 \\
\hline & 4 a 10 salários & 2 & 22,2 \\
\hline & $\geq 10$ salários & 0 & 0 \\
\hline & Não declarado & 1 & 11,1 \\
\hline
\end{tabular}




\begin{tabular}{|c|c|c|c|}
\hline Variável & Distribuição em categorias & $\mathbf{N}$ & $\%$ \\
\hline \multirow[t]{2}{*}{ Possui Religião } & Sim & 8 & 88,9 \\
\hline & Não & 1 & 11,1 \\
\hline \multirow[t]{2}{*}{ DCNT* } & Não & 8 & 88,9 \\
\hline & Sim & 1 & 11,1 \\
\hline
\end{tabular}

Fonte: elaborada pelos autores com base em dados da pesquisa. * Doenças Crônicas Não Transmissíveis.

Observou-se que a maioria dos participantes tinha baixa faixa salarial (até um salário mínimo/mês), assim como baixa renda familiar (1 a 3 salários mínimos/mês), considerando o número de moradores na casa da família (de 2 a 3 moradores). Em relação a esses achados, destaca-se que a existência de uma pessoa doente impõe a necessidade de gastos com transporte, compra de medicamento e outras demandas, sendo que tais gastos acabam comprometendo o já precário poder aquisitivo da família, especialmente quando a pessoa doente e/ou seu cuidador são os provedores da família ${ }^{(14)}$. Nesse sentido, acredita-se que as informações sobre a situação social e econômica da família das pessoas com câncer precisa ser incluída nas discussões da equipe de saúde, considerando as possibilidades de suporte e/ou encaminhar demandas específicas para serviços de apoio disponíveis.

A maioria dos participantes desse estudo informou possuir religião, dividindo-se entre a católica $(55,5 \%)$ e a evangélica (33,3\%). Sobre esse ponto, sabe-se que a crença em um ser superior, a fé, a espiritualidade e as práticas religiosas são importantes estratégias de enfrentamento da angústia, dando à pessoa em
$\mathrm{CP}$ e seus familiares conforto e sentido à vida, à dependência e ao cuidar ${ }^{(15)}$. Estudo sugere que, no que diz respeito à pessoa em $\mathrm{CP}$, mesmo não havendo uma crença religiosa instituída, a espiritualidade é expressa pelos pacientes, em especial por aqueles com proximidade do fim da vida. Assim, infere-se que negligenciar a espiritualidade seria descuidar de um componente essencial para as pessoas em $\mathrm{CP}^{(16)}$.

A espiritualidade dá esperança, propósito e significado para o processo de fim de vida da pessoa, possibilitando a criação de mecanismos para lidar com a doença, com as perdas, com a solidão e com a morte. Estudo apontou que o apoio espiritual fornecido pela equipe assistencial está associado a melhor QV, maior utilização dos CP e intervenções médicas menos agressivas no final de vida. Contudo, reconhece-se que esse tipo de apoio é pouco frequente entre os profissionais, devido a múltiplos fatores como a insuficiência de recursos humanos, falta de capacitação e escassez de tempo ${ }^{(17)}$.

Em relação à caracterização do perfil clínico, observou-se que o sítio primário do câncer mais comum entre os participantes foi o de mama $(n=3 ; 33,3 \%)$, como pode ser observado na Tabela 2 que se segue.

Tabela 2- Caracterização do perfil clínico dos pacientes em cuidados paliativos internados no setor de oncologia. Divinópolis, MG, mar.-jun., 2017 (n=9).

\begin{tabular}{|c|c|c|c|}
\hline Variável & Distribuição em categorias & $\mathrm{n}$ & $\%$ \\
\hline \multirow[t]{7}{*}{ Câncer Primário } & Mama & 3 & 33,3 \\
\hline & Cabeça/pescoço & 1 & 11,1 \\
\hline & Estômago & 1 & 11,1 \\
\hline & Colón/Reto & 1 & 11,1 \\
\hline & Pulmão & 1 & 11,1 \\
\hline & Útero & 1 & 11,1 \\
\hline & Próstata & 1 & 11,1 \\
\hline \multirow[t]{5}{*}{ Metástase primária } & Ossos & 4 & 44,4 \\
\hline & Fígado & 2 & 22,2 \\
\hline & SNC & 1 & 11,1 \\
\hline & Não declarado & 1 & 11,1 \\
\hline & Não possui & 1 & 11,1 \\
\hline \multirow[t]{2}{*}{ Realizou tratamento cirúrgico, em algum momento, no curso da doença? } & Sim & 5 & 55,6 \\
\hline & Não & 4 & 44,4 \\
\hline \multirow[t]{2}{*}{ Realizou radioterapia, em algum momento, no curso da doença? } & Sim & 6 & 66,7 \\
\hline & Não & 3 & 33,3 \\
\hline \multirow[t]{2}{*}{ Realizou quimioterapia, em algum momento, no curso da doença? } & Não & 7 & 77,8 \\
\hline & Sim & 2 & 22,2 \\
\hline \multirow[t]{2}{*}{ Realizou hormonioterapia, em algum momento, no curso da doença? } & Não & 6 & 66,6 \\
\hline & Sim & 3 & 33,4 \\
\hline
\end{tabular}


Como o câncer de mama é o tipo que mais acomete as mulheres no contexto nacional, é essencial que a equipe de enfermagem esteja preparada para atender às necessidades dessas pacientes em sua totalidade, de modo humanizado. Um estudo com mulheres com câncer de mama, em tratamento paliativo, apontou que as boas relações estabelecidas entre a mulher e o enfermeiro favorecem o processo de comunicação e cuidado, repercutindo na qualidade de vida da mulher ${ }^{(18)}$.

A maioria dos participantes do presente estudo apresentou uma metástase, sendo que os locais mais acometidos foram os ossos $(n=4$; $44,4 \%)$ e o fígado ( $n=2 ; 22,2 \%$ ). As metástases ósseas são, reconhecidamente, as metástases à distância mais frequentes relacionados ao câncer de mama. Além disso, as metástases nos ossos são frequentes nos cânceres avançados, ocorrendo em aproximadamente $50 \%$ dos pacientes com câncer metastático. Esse tipo de metástase causa elevada morbidade devido à dor, ao comprometimento da mobilidade, à hipercalcemia, às fraturas patológicas, dentre outros, repercutindo de forma importante na QV do paciente, pelas dores e as limitações físicas que impõem ${ }^{(19-20)}$. Já o fígado é considerado o principal órgão acometido por metástases hematogênicas provenientes da circulação portal advinda da circulação sistêmica. Esse fato pode ser atribuído ao fato de o fígado ser a maior víscera sólida do organismo, recebendo grande fluxo sanguíneo arterial e venoso ${ }^{(19)}$.

Os participantes desse estudo apresentavam histórico de tratamentos diversos ao longo do curso da doença, sendo que a maioria realizou tratamento cirúrgico $(n=5 ; 55,6)$ e radioterápico ( $n=6 \quad 66,7 \%$ ) em algum momento. Esse achado pode ter relação com a procura precoce pelos serviços de saúde e o diagnóstico oportuno da doença ${ }^{(14)}$.

A qualidade de vida dos participantes do estudo foi mensurada conforme indicação dos autores da FACIT-PAL, sendo que a análise dos dados por domínio encontra-se apresentada na Tabela 3.

Tabela 3 - Escores dos domínios da qualidade de vida dos pacientes oncológicos em Cuidados Paliativos. Divinópolis, MG, mar.-jun., 2017.

\begin{tabular}{lccccc}
\hline Estatística & $\begin{array}{c}\text { Bem-estar } \\
\text { Fésico }\end{array}$ & $\begin{array}{c}\text { Bem-estar } \\
\text { Social e familiar }\end{array}$ & $\begin{array}{c}\text { Bem-estar } \\
\text { Emocional }\end{array}$ & $\begin{array}{c}\text { Bem-estar } \\
\text { Funcional }\end{array}$ & $\begin{array}{c}\text { Preocupações } \\
\text { Adicionais }\end{array}$ \\
& $\mathbf{0 - 2 8}$ & $\mathbf{0 - 2 8}$ & $\mathbf{0 - 2 4}$ & $\mathbf{0 - 2 8}$ & $\mathbf{0 - 7 6}$ \\
Mediana & 16,0 & 15,0 & 11,0 & 12,0 & 34,0 \\
Mínimo & 5,0 & 4,0 & 2,0 & 5,0 & 29,0 \\
Máximo & 23,0 & 20,0 & 20,0 & 16,0 & 47,0 \\
Percentil 25 & 12,5 & 13,5 & 7,0 & 8,5 & 31,0 \\
Percentil 75 & 22,0 & 18,0 & 14,5 & 14,0 & 41,5 \\
\hline
\end{tabular}

Fonte: elaborada pelos autores com base em dados da pesquisa.

Pode-se observar, no presente estudo, que os domínios do instrumento de QV se comportaram de modo independente e autônoma, da mesma forma como aconteceu em estudo que também utilizou esse instrumento ${ }^{(21)}$.

De acordo com as orientações do grupo $F A C I T^{(12)}$, quanto maior o escore de pontuação nos diferentes domínios da escala, melhor é a QV. Os domínios que obtiveram os maiores escores foram o "bem-estar físico" (mediana=16; mínimo = 5; máximo= 23 pontos) e "bem-estar social-familiar" (mediana=15; mínimo= 4; máximo $=20$ pontos). Resultado semelhante foi identificado em estudo que utilizou a FACIT-Pal em pacientes sob cuidados paliativos, sendo que, nesse estudo, o domínio físico manteve as maiores pontuações em um seguimento de 18 meses desses pacientes ${ }^{(21)}$. Assim, pode-se inferir que a percepção dos participantes sobre sua condição física e seus relacionamentos sociais, assim como nos papéis sociais adotados na vida foram menos afetados por sua condição atual, pela doença e/ou pelo tratamento. Contudo, no mesmo estudo, mencionado anteriormente, participantes informaram que sintomas não contidos no instrumento de QV como a vertigem, instabilidade, dormências e confusão, associados às suas condições físicas e ao tratamento comprometeram sua capacidade de realizar tarefas simples da rotina de vida diária ${ }^{(21)}$.

Ao contrário do que foi identificado no presente estudo, o domínio de "bem-estar físico" obteve escores baixos nas primeiras quatro avaliações em pacientes com câncer avançado em terapêutica paliativa. Contudo, identificou-se melhores escores nesse domínio a partir da quinta avaliação, em um total de seis avaliações realizadas. Assim, os autores inferiram que houve 
melhor percepção de bem-estar no domínio físico com o progresso e continuidade da abordagem paliativa ${ }^{(10)}$.

Diante disso, entende-se que, apesar do avanço da doença e o tratamento comprometerem fisicamente o paciente, sua percepção sobre sua condição física pode ser positivamente influenciada pelo uso da terapêutica medicamentosa adequada para o controle dos sintomas, assim como pelo recebimento de suporte da equipe de saúde.

Apesar dos desafios impostos aos pacientes na sua capacidade de realizar as atividades de vida diária por suas limitações físicas, impostas pela doença e tratamento, estudo evidenciou que a aceitação da sua condição de saúde e a busca por ajudas para complementar as tarefas diárias auxiliaram os pacientes a superar as limitações ${ }^{(21)}$. Assim, salienta-se que a participação da rede de apoio dos pacientes em seus cuidados, assim como dos profissionais de saúde, são fundamentais para o enfrentamento dos pacientes dessa etapa da sua vida com qualidade.

Os escores do domínio de "bem-estar social-familiar" também obtiveram pontuações elevadas em outro estudo, sendo que os participantes desse apontaram que as interações sociais frequentes com familiares, amigos e profissionais de saúde foram essenciais para o recebimento do apoio necessário. A vivência da doença com os parceiros foi apontada por esses mesmos participantes como um reforçador da relação, sendo que o tempo gasto com os cuidados prestados pelos parceiros foram vistos como oportunidade de fortalecer e melhorar os relacionamentos ${ }^{(21)}$.

Outro estudo evidenciou que o "bem-estar social-familiar" foi bem avaliado por pacientes em tratamento paliativo nas primeiras avaliações realizadas com declínio nas que ocorreram nos últimos meses da pesquisa. Diante disso, os autores inferiram que esse domínio se torna mais presente e significativo com a evolução da doença, o que denota necessidade de intervenções específicas para a manutenção de adequado suporte social aos pacientes durante todo o curso da doença ${ }^{(10)}$.

Salienta-se que o controle de sintomas ainda continua sendo um desafio importante para a equipe de saúde que trabalha com pacientes com câncer avançado, considerando seu impacto direto sobre a QV e seu difícil manejo ${ }^{(20)}$.

As dimensões com maior prejuízo percebido entre os participantes desse estudo foi o "bem-estar emocional" (mediana= 11; mínimo= 2; máximo $=20$ pontos) e "bem-estar funcional" (mediana=12; mínimo $=5 ;$ máximo $=16$ pontos). Em estudo usando o mesmo instrumento de avaliação de QV também se obteve os piores escores no domínio emocional, sendo justificada pela percepção de mudanças importantes em seu dia a dia devido às suas condições de saúde ${ }^{(7)}$.

Outro estudo, observou-se pontuação elevada no domínio de bem-estar emocional, sendo que, nesse estudo, houve declínio na avaliação desse domínio apenas na avaliação realizada nos três meses que antecederam a morte do paciente. Emoções como tristeza, frustração, raiva e solidão foram expressados pelos participantes desse estudo, sendo que a manutenção da perspectiva positiva e busca por atividades de distração são apontadas como forma de superar emoções negativas ${ }^{(21)}$. 0 fornecimento de suporte emocional por parte da rede social do paciente e dos profissionais de saúde, principalmente nos meses que antecedem sua morte, pode favorecer o enfrentamento do paciente. Para tanto, é essencial que os profissionais da equipe assistencial sejam qualificados e desenvolvam a aptidão e sensibilidade necessárias à identificação de sinais não-verbais de necessidades emocionais que extrapolem necessidades informadas verbalmente pelo paciente.

Já em relação ao prejuízo percebido no "bem-estar funcional", considera-se que as limitações para o desenvolvimento das atividades de vida repercutiram na percepção de bem-estar dessas pessoas. Sabe-se que o declínio na funcionalidade, associado à progressão da doença, aos tratamentos e às comorbidades dos pacientes com câncer avançado é comum, sendo que autores indicam que os prejuízos na capacidade de participar de atividades habituais com pessoas significativas repercutem de modo importante na qualidade de vida ${ }^{(22)}$.

Resultado semelhante foi encontrado em estudo com mesmo instrumento, no qual os piores resultados foram obtidos no domínio "bem-estar funcional", sendo que os participantes desse, embora tentassem utilizar mecanismos de enfrentamento positivo ante ao seu estado de saúde, buscando aceitar suas limitações, alegaram que a constante necessidade de ajuda para realizar as atividades de vida diária impactou negativamente na sua percepção sobre sua capacidade funcional ${ }^{(21)}$. Nesse sentido, acredita-se que intervenções de enfermagem que 
favoreçam o autocuidado, respeitando as limitações e condições individuais dos pacientes em $\mathrm{CP}$, poderiam contribuir com a melhoria da percepção delas sobre sua capacidade funcional, possibilitando a melhoria na sua percepção em relação à QV.

$\mathrm{Na}$ avaliação dos índices globais do instrumento, obtiveram-se resultados que evidenciaram prejuízo na qualidade de vida dos participantes. No Índice do resultado do teste (Trial Outcome Index) obteve-se, como resultado, uma mediana de 62 pontos (mínimo $=52$; máximo=80) em 132 possíveis; no índice de qualidade de vida geral (FACT - G) obteve-se, como resultado, uma mediana de 53 pontos (mínimo=38; máximo=63) em 108 possíveis; já no índice total de qualidade de vida paliativa (FACITPal), obteve-se, como resultado, mediana de 84 pontos (mínimo $=76$; máximo $=110$ ) em 184 possíveis. Acredita-se que os fatos de que, na instituição em que os participantes desse estudo encontravam-se internados, inexista serviço de $\mathrm{CP}$, e os profissionais de saúde que atuam nesse contexto ainda têm uma aproximação bastante rasa com a temática, possam ter repercutido em definições tardias da necessidade dessa modalidade de cuidados e no retardo na implementação de medidas assistências com foco na QV. Estudo apontou que, como os CP são relativamente recentes no Brasil, um grande contingente de profissionais da saúde ainda desconhece essa modalidade de cuidado. Essa situação foi relacionada pelos autores à escassez de disciplinas voltadas ao ensino de conteúdos pertinentes à temática em currículos de cursos de graduação e pós-graduação no contexto nacional ${ }^{(23)}$.

Além disso, como os CP ainda se encontram em fase de consolidação no âmbito do sistema de saúde nacional, no município em que o presente estudo foi realizado, não existem serviços estruturados de prestação dessa modalidade de cuidados nos diferentes níveis de atenção, o que pode ter contribuído para a recepção tardia de cuidados com vistas ao conforto, a descontinuidade do cuidado e piora na percepção de bem-estar pelos pacientes. Em estudo recente que avaliou QV de pacientes em $C P$, pode-se identificar que o acompanhamento sistemático da equipe assistencial, por meio de visitas regulares e promoção de práticas paliativas mais precocemente, impactam positivamente na percepção da QV dos participantes $^{(21)}$.
Ressalta-se que 0 presente estudo apresenta limitações, principalmente relacionadas ao tamanho da amostra e seu delineamento descritivo, o que impossibilita estabelecer relação de causalidade. Além disso, salienta-se que os resultados encontrados não poderão ser generalizados para a população em geral, à medida que se trata de uma amostra por conveniência. Entretanto, acredita-se que os achados possam subsidiar a construção e condução de estudos futuros que avancem no sentido da avaliação da qualidade de vida de pacientes em cuidados paliativos, inclusive correlacionando-a com outras variáveis de interesse para essa população.

\section{CONCLUSÃO}

A QV dos pacientes internados em uma unidade de oncologia, mensurada por instrumento validado para esse fim, apresentou pontuações com discrepâncias na avaliação dos escores pelos participantes, que perceberam melhor bem-estar nos domínios referentes às dimensões física e social/familiar e pior bemestar nos domínios referentes às dimensões emocional e funcional. Essas informações denotam a necessidade de investimentos por parte da equipe de saúde e enfermagem em estratégias de identificação e resolução de questões que trazem prejuízos, tanto na capacidade de a pessoa em CP realizar suas atividades de vida diária como na sua forma de lidar e expressar as emoções que veem à tona e interferem na sua percepção sobre a QV.

Contudo, apesar de os escores nos domínios relacionados às dimensões física e social/familiar terem obtido melhor avaliação, é fundamental que a equipe de cuidado se instrumentalize para identificar precocemente sinais e sintomas de ordem física e inclua, no plano de cuidado, intervenções direcionados à manutenção do controle dos sintomas já existentes e a identificação e controle de novos sintomas. Além disso, é essencial que o enfermeiro avalie e monitore o suporte social e familiar fornecido ao paciente e provenha meios para que as pessoas que fazem parte da rede social do paciente participem ativamente de seu cuidado.

Ademais, entende-se ser necessária maior difusão dos conhecimentos sobre os CP entre os profissionais de saúde que atuam nos diferentes níveis de atenção, de modo que as práticas paliativistas possa fazer parte da assistência 
integral aos pacientes com doenças avançadas e fora de possibilidade terapêutica de cura.

\section{REFERÊNCIAS}

1. Barbosa IR, Costa ICC, Bernal MM, Souza DLB. Desigualdades socioeconômicas e mortalidade por câncer: Um estudo ecológico no Brasil. Rev Bras Promoç Saúde 2016 jul/set;29(3):350-6. DOI: 10.5020/18061230.2016.p350

2. Ministério da Saúde. Instituto Nacional de Câncer José Alencar Gomes da Silva. Estimativa 2016: Incidência de câncer no Brasil. Rio de Janeiro: Ministério da Saúde; 2015.

3. World Health Organization. Definição de cuidados paliativos. Geneva: WHO; 2005.

4. Freire MEM, Sawada NO, França ISX, Costa SFG, Oliveira CDB. Qualidade de vida relacionada à saúde de pacientes com câncer avançado: Uma revisão integrativa. Rev Esc Enferm USP 2014; 48(2):351-61. DOI: 10.1590/S0080-62342014000 $\underline{0200022}$

5. Maree JE, Van Rensburg JJMJ. Suitability of quality-of-life outcome measures in palliative care in the South African setting. Palliat Support Care. 2016 Apr;14:(2):118-28. DOI: 10.1017/S 1478951515000036

6. Siegert R, Selman L, Higginson IJ, Ali Z, Powell RA, Namisango $E$, et al. A psychometric evaluation of the Functional Assessment of Chronic Illness Therapy-Palliative Care (FACIT-Pal) scale with palliative care samples in three African Countrie. J Pain and Symptom Manage. 2014 Nov;48(5):983-91.

DOI:

10.1016/j.jpainsymman.2014. 01.010

7. Matos GDR, Pulschen AC. Qualidade de vida de pacientes internados em uma unidade de cuidados paliativos: Um estudo transversal. Rev Bras Cancerol. [Internet]. 2015 [acesso em 2017 nov 10];61(2):123-9. Disponível em: http://www. inca.gov.br/rbc/n 61/v02/pdf/06-artigo-

qualidade-de-vida-de-pacientes-internados-emuma-unidade-de-cuidados-paliativos-um-estudotransversal.pdf

8. Sawada NO, Nicolussi AC, Paula JM, Garcia CMP, Marti GC, Cruz QF. Quality of life of Brazilian and Spanish cancer patients undergoing chemotherapy: An integrative literature review. Rev Latino-Am Enfermagem 2016;24:e2688. DOI: 10.1590/1518-8345.0564.2688

9. Pereira FMP, Santos CSVB. Estudo de adaptação cultural e validação da Functional Assessment of Cancer Therapy-General em cuidados paliativos. Rev Enf Ref. 2011 dez;3(5): 45-54. DOI: 10.12707/RIII1041
10. Visentin A. Avaliação da qualidade de vida e sobrevida dos pacientes com câncer avançado na terapêutica paliativa [tese]. Curitiba: Universidade Federal do Paraná; 2016.

11. Ministério da Saúde. Portaria no 102, de 03 de fevereiro de 2012. Dispõe sobre as Unidades de Assistência de Alta Complexidade em Oncologia e dos Centros de Assistência de Alta Complexidade em Oncologia. Diário Oficial da União. 2012 fev.

12. Medeiros RB. Aplicação sequencial da escala de performance paliativa em pacientes paliativos internados em um hospital geral [dissertação]. Florianópolis: Universidade Federal de Santa Catarina; 2014.

13.Functional Assessment of Chronic Illness Therapy. Overview of the FACIT measurement system. Facit.org 2010. [acesso em 2017 nov 10]. Disponível em: http://www.facit.org/FACITOrg/ Overview

14. Souza RS, Simão DAS, Lima EDRP. Perfil sociodemográfico e clínico de pacientes atendidos em um serviço ambulatorial de quimioterapia paliativa em Belo Horizonte. Rev Min Enferm. 2012;16(1):38-47. DOI: S1415-2762220120001000 $\underline{06}$

15. Evangelista CB, Lopes MEL, Costa SFG, Batista PSS, Batista JBV, Oliveira AMM. Palliative care and spirituality: an integrative literature review. Rev Bras Enferm. 2016 jun;69(3):554-63. DOI: 10.1590/0034-7167.2016690324i

16. Mistretta EG. Spirituality in young adults with end-stage cancer: A review of the literature and a call for research. Ann Palliat Med. 2017 July;6(3): 279-83. DOI: $10.21037 / \mathrm{apm} .2017 .06 .17$

17. Balboni TA, Balboni M, Enzinge AC, Gallivan $K$, Paulk ME, Wrisht $A$, et al. Provision of spiritual support to patients with advanced cancer by religious communities and associations with medical care at the end of life. JAMA Intern Med. 2013 June;173(12):1109-17. DOI: 10.1001/jama internmed.2013.903

18. Cirilo JD, Silva MM, Fuly PSC, Moreira MC. A gerência do cuidado de enfermagem à mulher com câncer de mama em quimioterapia paliativa. Texto Contexto Enferm. 2016;25(3):1-9. DOI: 10.1590/0104-07072016004130015

19. Cheon PM, Pulenzas N, Zhang L, Mauti E, Wong $\mathrm{E}$, Thavarajah $\mathrm{N}$, et al. Fatigue scores in patients receiving palliative radiotherapy for painful bone metástases. Support Care Cancer. 2015 July;23(7)2097-2103. DOI: 10.1007/s00520014-2561-0

20. Currow DC, Clack K, Kamal A, Collier A, Agar $M R$, Lovel MR, et al. The population burden of 
chronic symptoms that substantially predate the diagnosis of a Life-Limiting Illness. J Palliat Med. 2015 jun;18(6):480-5. DOI: 10.1089/jpm.2014.04 $\underline{44}$

21. Frazer MS, Mobley P. A mixed methods analysis of quality of life among late-life patients diagnosed with chronic illnesses. Health Qual Life Outcomes 2017 Nov;15(1):222-31. DOI: 10.1186/s $\underline{12955-017-0797-3}$

22. Phillips JL, Lam L, Luckett T, Agar M, Currow D. Is the life space assessment applicable to a palliative care population? Its relationship to measures of performance and quality of life. J Pain Symptom Manage. 2014 June;47(6):1121-7. DOI: 10.1016/j.jpainsymman.2013.06.017

23. Hermes HR, Lamarca ICA. Cuidados paliativos: Uma abordagem a partir das categorias profissionais de saúde. Ciênc. saúde coletiva. 2013 set;18(9):2577-88. DOI: 10.1590/S1413-81 $\underline{232013000900012}$

Nota: Trabalho de pesquisa proveniente do Trabalho de Conclusão de Curso desenvolvido por alunos do Curso de graduação em Enfermagem da Universidade Federal de São João del-Rei - UFSJ.

Recebido em: 21/12/2017

Aprovado em: 04/06/2018

Endereço de correspondência:

Raissa Silva Souza

Av. Vinte e um de Abril, 429, Casa 02 - Centro

CEP: 35500-010 - Divinópolis/MG - Brasil

E-mail: rssouza.ra@ufsj.edu.br 